Bundesgesundheitsbl 2019 · 62:232-234

https://doi.org/10.1007/s00103-018-2870-4

(c) Springer-Verlag GmbH Deutschland, ein Teil von Springer Nature 2018

Bekanntmachung des Umweltbundesamtes

\title{
Mögliche Auswirkungen des Klimawandels auf die Luftqualität in Innenräumen
}

\author{
Stellungnahme der Kommission \\ Innenraumlufthygiene (IRK) am \\ Umweltbundesamt
}

Der ungewöhnliche Sommer des Jahres 2018 mit sehr heißen und trockenen Perioden, unterbrochen von Starkregen, hat die möglichen Konsequenzen einer Klimaveränderung erneut deutlich vor Augen geführt. Besondere Gesundheitsrisiken durch steigende Temperaturen werden von Watts et al. [1] für die Bevölkerung in Mitteleuropa und im östlichen Mittelmeergebiet gesehen. In solchen heißen Sommern werden vielerorts sowohl im privaten Bereich als auch an Arbeitsplätzen intensive Maßnahmen zum Schutz vor lang andauernden hohen Temperaturen erforderlich. Als Reaktion auf die zunehmenden Extremwetterereignisse hat die Bundesregierung im Jahr 2008 die Nationale Anpassungsstrategie an den Klimawandel (DAS) etabliert. In diesem Kontext hat das Bundesumweltministerium bereits vor einigen Jahren das Kompetenzzentrum Klimafolgen und Anpassung (KomPass) am Umweltbundesamt errichtet, welches die Folgen des Klimawandels genauer untersuchen und Strategien zur Klimaanpassung erarbeiten soll.

Aktuell veröffentlichte die Bundesregierung das Deutsche Klimavorsorgeportal, das konkret für den Bereich Innenraumluft derzeit die beiden Vorsorgedienste „DWD-Hitzewarnung“ und „Arbeitsstättenregel ASR A3.5 Raumtemperatur" anbietet (siehe: https://www.klivoportal.de/DE/Home/home_node.html; -> Dienste -> Handlungsfeld -> menschl. Gesundheit -> suche: Innenraum).

Die Auswirkungen des Klimawandels können die Luftqualität in Innenräumen negativ beeinflussen. Besonders unter präventiven Gesichtspunkten ist es daher von Bedeutung, diesen Aspekt bei Bauvorhaben und Wohnkonzepten zu berücksichtigen. Dabei sind sowohl bauliche Maßnahmen (passiver Wärmeschutz) als auch Veränderungen im Lüftungsverhalten im Sommer und/oder der Einbau von Lüftungstechniken (aktiver Wärmeschutz) zu empfehlen.

\section{Einfluss des Außentemperaturanstiegs auf Innenraumklima und Raumluftqualität}

Die Auswirkungen von Hitzeperioden auf die menschliche Gesundheit sind gut dokumentiert $[2,3]$. Deswegen ist auch die Gefahr übermäßiger Aufheizung von Innenräumen mit entsprechenden Folgen für die Innenraumluftqualität und das Wohlbefinden ein wichtiger Faktor in der öffentlichen Diskussion.

Klimatologisch bezeichnet man einen Tag mit einer Höchsttemperatur von mindestens $25^{\circ} \mathrm{C}$ als „Sommertag“ und einen Tag mit einer Höchsttemperatur von mindestens $30^{\circ} \mathrm{C}$ als „heißen Tag“ [4]. Allgemein ist bei Raumlufttemperaturen oberhalb von $26^{\circ} \mathrm{C}$ körperliches und geistiges Arbeiten und Lernen nur eingeschränkt möglich, da Konzentration und Aufmerksamkeit nachlassen. Für Büroräume sieht die Technische Regel ASR A 3.5 zur Arbeitsstättenverordnung daher ab einer Innenraumlufttemperatur von $26^{\circ} \mathrm{C}$ personenbezogene Maßnahmen und $\mathrm{ab}$ einer
Raumlufttemperatur von $30^{\circ} \mathrm{C}$ organisationsbezogene Maßnahmen vor. Ältere und empfindliche Personen leiden besonders unter den hohen Temperaturen. Mögliche Maßnahmen bei Hitzeereignissen sind in den Handlungsempfehlungen (Kernelement 4 „Reduzierung von Hitze in Innenräumen“) für die Erstellung von Hitzeaktionsplänen zum Schutz der menschlichen Gesundheit der Bund/Länder Ad-hoc Arbeitsgruppe „Gesundheitliche Anpassung an die Folgen des Klimawandels" [5] aufgeführt.

In Innenräumen spielt neben der Raumlufttemperatur die relative Luftfeuchtigkeit eine wichtige Rolle. Sind beide Faktoren hoch, verstärkt dies in der Regel auch die Freisetzung chemischer Stoffe, z. B. aus Baustoffen oder Einrichtungsgegenständen, und führt somit $\mathrm{zu}$ höheren Konzentrationen flüchtiger organischer Verbindungen in der Raumluft. Konstant hohe Außenlufttemperaturen in den Sommermonaten bewirken zwangsläufig auch erhöhte Temperaturen der Massivbauteile eines Gebäudes. Insbesondere in der älteren Gebäudesubstanz sind nicht selten als kritisch einzustufende Baustoffe vorhanden. Die Wahrscheinlichkeit von Richtwert überschreitenden Gefahrstoffbelastungen in Innenräumen steigt mit der Zunahme der Temperatur.

Generell kann Luft bei höheren Temperaturen mehr absolute Feuchtigkeit aufnehmen als kältere Luft. Im Sommer, wenn die Außenluft nicht nur warm sondern auch feucht (schwül) ist, gelangt absolut gesehen viel Luftfeuchte in den In- 
nenraum. In Altbauten, vorwiegend in Kellerräumen, Souterrainwohnungen mit Außenwänden, die teils unter der Geländeoberkante liegen, oder auch in historischen Gebäuden mit massiven sehr dicken Wänden, z.B. in Kirchen, kann dies zur Folge haben, dass sich die relative Luftfeuchtigkeit im Innenraum entlang kalter Wände schlagartig erhöht und das Risiko für Schimmelbefall steigt [6]. Dies ist nicht primär eine Folge des Klimawandels; die Situation kann dadurch aber noch verschärft werden.

\section{Baulicher Wärmeschutz}

Gebäude sollten zukünftig verstärkt so geplant und gebaut werden, dass sie neben dem erforderlichen winterlichen Wärmeschutz auch im Sommer vor Gebäudeaufheizung schützen. Dies sollte vor allem bei großflächiger Ausrichtung von Fenstern zur Südseite, durch passive Maßnahmen wie außenliegenden Sonnenschutz, und die Möglichkeit der Nachtauskühlung erreicht werden. Passive Solargewinne über z.B. Fensterflächengestaltungen, die in den Übergangsmonaten helfen, die Energiebilanz zu verbessern, sind im Hochsommer durch Beschattungsmaßnahmen $\mathrm{zu}$ vermeiden.

Bei Gebäuden mit Lüftungstechnik ist die Kühlung im Sommer technisch möglich, aber nicht das vorrangige Ziel [7]. Im Hochsommer sollte das Lüften bevorzugt in die späten Abend- oder frühen Morgenstunden verlegt werden. Eine Querstrom- oder Durchzugslüftung kann helfen, den Luftaustausch zu beschleunigen. Allerdings kann es bei längerem Durchzugslüften zu unangenehmen Zugerscheinungen kommen. Oftmals reicht aber auch Durchzugslüftung allein nicht aus, um der Gebäudeaufheizung im Hochsommer dauerhaft entgegenzuwirken.

\section{Auswirkungen energieeffizienter Maßnahmen an Gebäuden}

Um unnötige Wärmeverluste zu vermeiden und die gültigen energetischen Standards einzuhalten, werden Gebäude (luft) dicht gebaut bzw. saniert. Dies bedeutet, dass in Gebäuden ohne raumlufttechnische Anlagen die Nutzer gefordert sind, ausreichend zu lüften, um den erforderlichen Luftwechsel sicherzustellen. Im Sommer kann das Lüften zum falschen Zeitpunkt aber zur weiteren Aufheizung führen und bedingt - je nach Wetterlage - das Risiko des Eintrags warmer, feuchter und ggf. auch schadstoffreicher Luft von außen. Im Jahr 2018 wurden besonders in den Monaten Juli und August vielerorts erhöhte Belastungen der Außenluft mit Ozon, Stickoxiden, Feinstaub und anderen Stoffen registriert (https://www. umweltbundesamt.de/daten/luft/ozonbelastung). An solchen Tagen empfiehlt es sich, Fenster und Türen tagsüber geschlossen zu halten [8]. Darüber hinaus kann der Eintrag von Ozon und Stickoxiden beim Lüften zu chemischen Reaktionen im Innenraum und damit zur Bildung sogenannter Sekundärprodukte führen, über deren gesundheitliche Risiken nicht immer klare Erkenntnisse vorliegen. Oft handelt es sich dabei um reaktive Oxidationsprodukte ungesättigter Kohlenwasserstoffe, die darüber hinaus zu organischen Aerosolen kondensieren können [9]. Auch deswegen empfiehlt es sich, das Lüften im Hochsommer in die Abendstunden zu verlegen.

\section{Lüftungsanlagen}

Bereits seit einiger Zeit wird diskutiert, bei neuen bzw. aufwendig sanierten älteren Gebäuden zur Sicherstellung guter Innenraumluftqualität lüftungstechnische Anlagen zu installieren. In Passiv- und Nullenergiehäusern ist der Einbau von lüftungstechnischen Anlagen mit Wärmerückgewinnung bereits beschriebener Standard [7]. In allen anderen energiebedarfsarmen Gebäuden ist der Einbau zu empfehlen, bei nicht energiebedarfsarmen Gebäuden im (Alt)bestand sind differenzierte Maßnahmen möglich [10]. Derzeit verfügen Lüftungsanlagen in Wohngebäuden aus Kostengründen und wegen erhöhten technischen Aufwands auch bei der späteren Wartung nur selten über geregelte Einrichtungen zur Luftkühlung (Klimatisierung).Transportable Geräte zur Luftkühlung (mobile Klimageräte) sind aus Sicht der IRK nur als vorübergehende Maßnahme sinnvoll, um die Raumlufttemperaturen kurzfristig zu senken [11]. Solche Geräte benötigen eine regel- mäßige Reinigung und Wartung, da ansonsten eine Verkeimungsgefahr besteht. Sie verbrauchen zudem beim Betrieb oft erhebliche Mengen an elektrischer Energie.

\section{Emissionsarme Bauprodukte}

Temperaturerhöhung fördert in der Regel die Freisetzung von flüchtigen Verbindungen aus Bauprodukten und Einrichtungsgegenständen. Emissionsarme Produkte leisten daher einen wesentlichen Beitrag für eine bessere Luftqualität im Innenraum, auch im Hinblick auf energieeffiziente Gebäude [12]. Dies gilt auch und im Besonderen bei zusätzlichen Emissionen in überhitzten und feuchten Räumen. Daher sollte künftig verstärkt auf die Verwendung emissionsarmer Produkte, die u. a. am Blauen Engel zu erkennen sind, in Bau- und Ausstattungsphase geachtet werden. Bei der Ableitung von Bewertungskriterien für Produktemissionen wird es ggf. notwendig sein, zukünftig auch eine höhere Temperaturbeanspruchung zu berücksichtigen.

Im Einsatz luftreinigender Geräte sieht die IRK keine Alternative zu emissionsarmen Produkten [11].

\section{Literatur}

1. Watts N, Amann M, Arnell N et al (2018) The 2018 report of the Lancet Countdown on health and climate change: shaping the health of nations for centuries to come. Lancet. https:// doi.org/10.1016/S0140-6736(18)32594-7

2. Steul K, Schade M, Heudorf U (2018a) Mortality during heatwaves 2003-2015 in Frankfurt-Main the 2003 heatwave and its implications. Int J Hyg Environ Health 221:81-86

3. Steul K, Latasch L, Jung H-G, Heudorf U (2018b) Morbidität durch Hitze - eine Analyse der Krankenhauseinweisungen per Rettungseinsatz während einer Hitzewelle 2015 in Frankfurt/Main. Gesundheitswesen 80:353-359

4. Brasseur GP, Jaco D, Schuck-Zöller S (Hrsg) (2017) Klimawandel in Deutschland. Springer Spektrum, Berlin

5. Bund/Länder Ad-hoc Arbeitsgruppe "Gesundheitliche Anpassung an die Folgen des Klimawandels $(G A K)^{\prime \prime}$ (2017) Handlungsempfehlungen für die Erstellung von Hitzeaktionsplänen zum Schutz der menschlichen Gesundheit. Bundesgesundheitsblatt 60:662-672

6. Umweltbundesamt (2017) Leitfaden zur Vorbeugung, Erfassung und Sanierung von Schimmelbefall in Gebäuden. Umweltbundesamt, DessauRoßlau. 
7. Arbeitskreis Lüftung am Umweltbundesamt (2018) Anforderungen an Lüftungskonzeptionen in Gebäuden - Teil l: Bildungseinrichtungen. Bundesgesundheitsblatt 61:239-248

8. Salthammer T, Schieweck A, Gu J, Ameri S, Uhde E (2018) Future trends in ambient air pollution and climate in Germany - Implications for the indoor environment. Build Environ 143:661-670

9. Morrison $\mathrm{G}$ (2010) Chemical reactions among indoor pollutants. In: Lazaridis M, Colbeck I (Hrsg) Human Exposure to Pollutants via dermal Absorption and Inhalation. Springer, Dordrecht, S 73-96

10. Arbeitskreis Lüftung am Umweltbundesamt (2019) Anforderungen an Lüftungskonzeptionen in Gebäuden - Teil II:Wohngebäude. Bundesgesundheitsblatt in Vorbereitung

11. IRK (2015) Stellungnahme der Innenraumlufthygiene-Kommission (IRK) am Umweltbundesamt zu Luftreinigern. Bundesgesundheitsblatt 58:1192

12. Müller B, Mertes A, Scutaru AM (2016) Innenraumluftqualität nach Einbau von Bauprodukten in energieeffizienten Gebäuden. Texte, Bd. 36. ISSN: 1862-4804. Umweltbundesamt, Dessau-Roßlau 